\title{
Analisis de la gestión del liderazgo y valores en la administración de las unidades educativas.
}

\author{
Analysis of the use of a heater to electromagnetic induction to provide hot \\ water for single-family homes.
}

Jorge Luis Puyol Cortez. MSc. ${ }^{1}$, Evelyn Eugenia Alcívar Soria. MAE. ${ }^{2}$, López Martha Cecilia. MAE. ${ }^{3}$, Ramiro Enrique Guamán Chávez. MSc. ${ }^{4}$

\begin{abstract}
.
DOI: https://doi.org/10.33262/cienciadigital.v2i1.15

The purpose of this work is to evaluate the management, leadership and values in the administration of the "Domingo Savio" Fissional School of the city and province of Esmeraldas, aiming to solve the problem that is presented in the institutional planning documents "the non-inclusion in the instruments of educational management of complementary scientific, cultural, artistic and recreational training activities for an integral formation of the students ", who occupy their free time in unproductive activities. Qualitative and quantitative research was carried out, of an exploratorydescriptive type, using the survey technique, to obtain results and after the discussion.
\end{abstract}

Keywords: Educational Management, Education Administration, Leadership,

Education

\section{Resumen.}

Este trabajo tiene como finalidad evaluar la gestión, liderazgo y valores en la administración del Colegio Fiscomisional "Domingo Savio" de la ciudad y provincia de Esmeraldas, apuntando a solucionar el problema que se presenta en los documentos de planificación institucional " la no inclusión en los instrumentos de gestión educativa de actividades de formación complementarias científicas,

\footnotetext{
${ }^{1}$ Universidad Técnica Luis Vargas Torres de Esmeraldas Ext. la Concordia,jpuyol173@gmail.com

${ }^{2}$ Universidad Técnica. Luis Vargas Torres de Esmeraldas Ext. la Concordia, evelyn_alcivar@hotmail.com

${ }^{3}$ Universidad Técnica Luis Vargas Torres de Esmeraldas Ext. la Concordia, cecilia.lopez@utelvtg.edu.ec

${ }^{4}$ Universidad Técnica Luis Vargas Torres de Esmeraldas Ext. la Concordia, ramiro.guaman@utelvt.edu.ec
} 
culturales, artísticas y recreativas para una formación integral de los estudiantes", quienes ocupan su tiempo libre en actividades poco productivas. Se realizó una investigación cualitativa y cuantitativa, de tipo exploratorio-descriptivo, utilizando la técnica de la encuesta, para obtener resultados y posterior a la discusión.

Palabras Claves: Gestión educativa, administración de la educación, liderazgo, educación.

\section{Introducción.}

La investigación sobre gestión curricular y valores pone de manifiesto consistentemente la importancia del liderazgo de la dirección de todo centro escolar. Seguro de que estos elementos permiten alcanzar un clima propicio entre los miembros de la comunidad educativa, me permito investigarlos en el centro de educación general básica "Domingo Savio", esta institución Educativa nace por una necesidad imperiosa de la comunicad lo que a su vez se complementó con el proceso educativo realizado por las escuelas Fisco-misionales Sagrado Corazón y Don Bosco de la ciudad de Esmeraldas ya que este quedaba inconcluso cuando los alumnos que terminaban la instrucción primaria se dispersaban a diferentes Colegios de la ciudad (Amat, 2007).

Para asegurar continuidad del trabajo en la consecución del objetivo educativo, humano, solidario, único y trascendente; se vio la necesidad de crear un Centro de educación básica que acoja a todos los estudiantes y continúe el proceso. Partiendo de la importancia de la dirección en el funcionamiento de los centros educativos, de su rol fundamental en el tipo y logro de los objetivos, en el clima de relaciones, en el dinamismo en que muchas de las escuelas están inmersas como organizaciones que aprenden, se pretende con este trabajo aportar con el análisis del liderazgo y el clima escolar de los centros educativos de nivel medio de la localidad, destacando algunos aspectos fundamentales relacionados con la dirección de los mismos, además de identificar las falencias y debilidades de estos en cuanto a la educación en valores, conscientes de que un tratamiento del tema en profundidad precisaría de mucha más dedicación y espacio (Aguerrondo, 1991). Para esto se tomó en cuenta el tipo de dirección que se debe realizar en la educación actual, las formas de gestión de los Recursos Humanos, centradas en las personas, la gestión del clima escolar, la importancia de la gestión del conocimiento, de la comunicación en los centros, el trabajo en equipo y en red, la importancia del liderazgo educativo y docente, y la implantación de planes de calidad se analizan desde la perspectiva del rol de la dirección para dar respuesta a los retos (Calderón, 2015).

El presente trabajo investigativo propone describir los principios fundamentales y teóricos en lo referente a "Gestión en liderazgo y valores" en la Centro de Educación General Básica 
"Domingo Savio", analizando su situación actual la misma que aparentemente es idónea, pero en la realidad el clima escolar necesita mejorar para que la institución sea un verdadero referente educativo dentro de la provincia y por ende del país. Se toma en cuenta el sentir de todos los actores educativos, los mismos que están involucrados de forma directa con el proceso y puedan dar orientación del funcionamiento de todos y cada uno de los departamentos del centro (Castro , 2014).

Se puede resaltar que una investigación de la trascendencia que esta tiene no se ha realizado aún en el centro educativo, con este antecedente resalto la importancia de tratar este tema en un centro de esta naturaleza ya que el clima escolar, el liderazgo administrativo y el liderazgo educacional basado en valores forman parte de los elementos básicos para una educación de calidad. Se demuestra la factibilidad de este trabajo gracias a la existencia de los documentos curriculares del centro, las guías de educación en valores y liderazgo emitidas por la UTPL, las cuales permitieron comparar el funcionamiento del centro con los modelos ideales propuestos por los autores, junto con la colaboración de los directivos, profesores, padres de familia y estudiantes del centro educativo quienes deseaban una oportunidad como esta para provocar un cambio positivo en el funcionamiento de la gestión educativa en la institución, es importante resaltar que los actores de este centro educativo respondieron honestamente a cada una de las interrogantes planteadas, lo que facilitó el trabajo ya que en la verificación in situ se constató la realidad de lo respondido y la existencia o no de la documentación curricular necesaria (Fernandez, Morales, \& Portal, 2004).

El trabajo de campo se lo realizó al tomar en cuenta a toda la muestra poblacional propuesta conformada por 4 directivos, 20 docentes, 20 estudiantes y 20 padres de familia a los mismos que se les realizó las respectivas encuestas y entrevistas, a más de observar y estudiar todos los documentos relacionados a gestión curricular, esto permitió conocer más a fondo la problemática del centro y recomendar acerca de cómo fortalecer la gestión para la obtención de un clima escolar idóneo. Es importante saber que los objetivos tanto general como específicos nos avizoran un amplio camino de opciones por descubrir e investigar, estos trazan la meta hacia la formación de verdaderos líderes tanto estudiantiles como docentes, resaltando el valor que tiene la parte axiológica del ser humano ya que los valores hacen de la persona un ente positivo en cualquier medio que este se desenvuelva (Guachamin, 2012).

Todos estos aspectos convierten a este trabajo en único en su género en la institución y en todas las instituciones similares en el medio, lo que lo vuelven interesante y de buena aceptación, ya que su aplicación permitirá tener centros educativos eficientes y de gran aceptación por la comunidad educativa en general, es por ello que se pondrá a disposición de todos los directivos de los colegios fiscomisionales de Esmeraldas quienes tendrán la oportunidad de estudiarla y aplicarla. La Gestión Educativa se compone de tres dimensiones: 
La pedagógica y didáctica, la administrativa, y la socio - humanística o comunitaria, cuyo principio base es la participación de manera colectiva, para lograr involucrar, concientizar y por lo tanto consensuar, y así alcanzar los resultados planeados y deseados (Morales , 2012)

Por lo tanto, la gestión educativa consiste en:

- Presentar un perfil integral, coherente y unificado de decisiones.

- Definir los objetivos institucionales, las propuestas de acción y las prioridades en la administración de recursos.

- Definir acciones para extraer ventajas a futuro; se consideran tanto las oportunidades y amenazas del medio en el que está inserta, como los logros y problemas de la misma organización.

- Comprometer a todos los actores institucionales. Definir el tipo de servicio educativo que se ofrece.

La Gestión ocurre en todos los espacios o áreas componentes de la comunidad educativa institucional, local, regional o nacional, y la misma pasa por momentos de diagnóstico, planeación, ejecución, seguimiento y evaluación que se nutren entre sí y conducen a la obtención de los resultados definidos por los equipos directivos. Fundamentalmente, la gestión ocurre en los establecimientos escolares que es donde se desarrollan los procesos de enseñanza, aprendizaje y convivencia entre los diferentes actores; es también el lugar donde se materializan el Proyecto Educativo Institucional (PEI o PEC), el currículo y el plan de estudios, y se concretan la planeación curricular, las actividades pedagógicas, las evaluaciones y autoevaluaciones y las relaciones con diferentes interlocutores de la comunidad educativa y otras entidades (Morales, 2012).

La Gestión Educativa, denominada también Gestión de procesos, toma en cuenta cómo la organización escolar desarrolla de manera sistemática e intencional los sub- procesos institucionales que, en sus diversas dimensiones, se implementan con el fin de alcanzar resultados de calidad, tales como la gestión curricular y pedagógica, el desarrollo de los procesos regulares del establecimiento y el soporte administrativo de la gestión de la enseñanza; y así mismo, la forma en que se evidencia la preocupación por el mejoramiento continuo y la promoción de la búsqueda de soluciones innovadoras a los problemas de gestión (Palacios , 2011).

Destaca como importancia de la gestión educativa que es un instrumento articulador de la gestión pedagógica, administrativa y de recursos en donde el nivel del aula debe ser apoyado por los niveles superiores de la organización escolar. Los equipos directivos de las escuelas son actores clave de estos procesos de organización escolar porque son los responsables de promover nuevas culturas de trabajo apoyadas en el funcionamiento de equipos docentes, en 
la construcción de redes con otras escuelas y otras instituciones de la comunidad y en el diseño, desarrollo y evaluación de propuestas de enseñanza consensuadas (Peréz , 2014).

La gestión educativa también toma importancia ya que influye directamente en los resultados educativos de los alumnos, esto quiere decir que un establecimiento que mejore de manera significativa su gestión escolar, mejorará también los resultados académicos de sus alumnos, en añadidura, la gestión escolar ayuda al trabajo educativo porque permite lograra buenos resultados aún con alumnos de condiciones socioeconómicas desmejoradas y alta vulnerabilidad educativa, es decir, es un factor de equidad en la educación (Quintana \& Moreno, 2007).

Llevar a cabo una verdadera gestión educativa tiene la ventaja que no implica mayor gasto, es decir que con los pocos recursos que cuente una institución se puede obtener buenos resultados. Cobra importancia porque permite mejorar el clima de trabajo, tanto directivos como docentes, lo que permite mantener un centro educativo más comprometido y motivado con la educación de calidad y la formación integral de los educandos (Rodriguez \& Bravo, 1991).

\section{Métodos y materiales.}

Esta investigación se realizó al Centro Educativo Fisco misional de Educación General Básica "Domingo Savio", para el desarrollo de este proceso investigativo es el método descriptivo, exploratorio y deductivo- analítico c y la aplicación de una encuesta al personal administrativo, docentes estudiantes y padres de familia.

Como la investigación de campo consiste en recoger los datos de interés en forma directa y al ser parte de la institución educativa me permitió iniciar el proceso solicitando el debido permiso documentado a los directivos y a la vez realizar el cronograma de acciones a realizar como son las encuestas, entrevistas y análisis de los documentos solicitados, para las entrevistas a los padres se solicitó por parte de la autoridad respectiva una reunión especial donde participaron el $100 \%$ de padres invitados.

Una vez realizada la investigación se procede a clasificar y procesar los datos en tablas estadísticas para luego presentarlos en diagramas los que permiten una visualización más detallada y facilita su respectivo análisis, el mismo que será de vital importancia para la detección del problema a solucionar sobre la base de una crítica teórica y fundamentada plantear la propuesta de solución que será presentada a las autoridades del centro con el fin de mejorar el desempeño en valores y liderazgo de la misma.

\section{Análisis de resultado.}

El centro de educación general básica "Domingo Savio, es una institución Fiscomisional católica, ubicada en la zona urbana de la ciudad y provincia de Esmeraldas, es un establecimiento que cumple con la exigencia del Ministerio de educación de ser escuela completa de 10 grados; se inspira en la enseñanza del Evangelio y en el espíritu Salesiano, tomando como base la pedagogía de San Juan Bosco conocida como "Sistema Preventivo" cuyos elementos fundamentales son: la razón, la religión y la amabilidad ofreciendo así una educación popular de calidad. 
TABla 1. De la EnCUesta A los DOCENTES

\begin{tabular}{|c|c|c|c|c|c|c|c|}
\hline \multirow{2}{*}{ Orden } & \multirow{2}{*}{ Declaraciones } & \multicolumn{2}{|c|}{ Siempre } & \multicolumn{2}{|c|}{ A veces } & \multicolumn{2}{|c|}{ Nunca } \\
\hline & & f & $\%$ & f & $\%$ & f & $\%$ \\
\hline 1 & $\begin{array}{l}\text { El rol del docente líder se define como una persona que posee } \\
\text { la actitud y las habilidades para cuestionar las órdenes } \\
\text { existentes }\end{array}$ & 2 & 10 & 14 & 70 & 4 & 20 \\
\hline 2 & $\begin{array}{l}\text { El liderazgo en la unidad educativa está intrínseca búsqueda } \\
\text { de la innovación y el cambio a través de la transformar las } \\
\text { formas habituales de la escolarizar }\end{array}$ & 12 & 60 & 8 & 40 & 0 & 0 \\
\hline 3 & $\begin{array}{l}\text { La gerencia educativa se, promueve en los padres, } \\
\text { representantes, comunidad en general la importancia de } \\
\text { brindar a los estudiantes un ambiente de aprendizaje } \\
\text { agradable, armónico, seguro y estimulante }\end{array}$ & 16 & 80 & 4 & 20 & 0 & 0 \\
\hline 4 & $\begin{array}{l}\text { Los directivos y docentes promueven la investigación a nivel } \\
\text { educativo porque es un producto de la participación colectiva } \\
\text { donde se integran docentes- estudiantes- familias- asociación } \\
\text { civil- padres y representantes- consejo comunal con el fin de } \\
\text { desarrollar y materializar metas del centro educativo. }\end{array}$ & 10 & 50 & 9 & 45 & 1 & 5 \\
\hline 5 & $\begin{array}{l}\text { Resistencia o escepticismo en los padres cuando se intenta } \\
\text { llevar a cabo nuevos métodos de enseñanza }\end{array}$ & 2 & 10 & 13 & 65 & 5 & 25 \\
\hline 6 & $\begin{array}{l}\text { Trabajo en equipo, para tomar de decisiones de cambio de } \\
\text { metodologías de enseñanza aprendizaje }\end{array}$ & 13 & 65 & 7 & 35 & 0 & 0 \\
\hline 7 & $\begin{array}{l}\text { En el proceso de enseñanza aprendizaje los valores es el eje } \\
\text { trasversal de la formación integral del estudiante }\end{array}$ & 16 & 80 & 4 & 20 & 0 & 0 \\
\hline 8 & $\begin{array}{l}\text { Resistencia en los compañeros o director/rector cuando } \\
\text { intento desarrollar nuevos métodos de enseñanza }\end{array}$ & 2 & 10 & 7 & 35 & 11 & 55 \\
\hline 9 & $\begin{array}{l}\text { Sentirme poco integrado en la escuela y entre los } \\
\text { compañeros }\end{array}$ & 5 & 25 & 2 & 10 & 13 & 65 \\
\hline 10 & $\begin{array}{l}\text { Desacuerdo continuo en las relaciones con el director del } \\
\text { centro educativo. }\end{array}$ & 2 & 10 & 7 & 35 & 11 & 55 \\
\hline 11 & Admiro el liderazgo y gestión de las autoridades educativas & 7 & 35 & 9 & 45 & 4 & 20 \\
\hline 12 & $\begin{array}{l}\text { Me siento comprometido con las decisiones tomadas por el } \\
\text { Director/Rector del centro educativo. }\end{array}$ & 12 & 60 & 6 & 30 & 2 & 10 \\
\hline 13 & $\begin{array}{l}\text { Los directivos mantienen liderazgo y gestión en el área } \\
\text { académica }\end{array}$ & 8 & 40 & 9 & 45 & 3 & 15 \\
\hline 14 & $\begin{array}{l}\text { Los directivos mantiene liderazgo y gestión en el área } \\
\text { administrativa-financiera }\end{array}$ & 9 & 45 & 9 & 45 & 2 & 10 \\
\hline 15 & $\begin{array}{l}\text { Actividades de integración en los ámbitos deportivo y } \\
\text { sociocultural con la participación de autoridades, padres de } \\
\text { familia, docentes y estudiantes }\end{array}$ & 17 & 85 & 3 & 15 & 0 & 0 \\
\hline 16 & $\begin{array}{l}\text { Los valores predominan en las decisiones de los directivos } \\
\text { y profesores }\end{array}$ & 15 & 75 & 5 & 25 & 0 & 0 \\
\hline
\end{tabular}

Fuente: Encuesta a docentes, Centro Educativo "Domingo Savio"

Elaborado Por: Grupo de Investigación 
Tabla 2. De la encuesta a estudiantes Tabla 29

\begin{tabular}{|c|c|c|c|c|c|c|c|}
\hline \multirow{2}{*}{ Orden } & \multirow{2}{*}{ Declaraciones } & \multicolumn{2}{|c|}{ Siempre } & \multicolumn{2}{|c|}{ A veces } & \multicolumn{2}{|c|}{ Nunca } \\
\hline & & $\mathbf{f}$ & $\%$ & $\mathbf{f}$ & $\%$ & $\mathbf{f}$ & $\%$ \\
\hline 1 & $\begin{array}{l}\text { El Director/Rector tiene en cuenta las opiniones de los } \\
\text { docentes y estudiantes }\end{array}$ & 13 & 65 & 5 & 25 & 2 & 10 \\
\hline 2 & $\begin{array}{l}\text { Las autoridades hablan más que escuchanlos problemas de } \\
\text { los estudiantes }\end{array}$ & 3 & 15 & 14 & 70 & 3 & 15 \\
\hline 3 & $\begin{array}{l}\text { El liderazgo conductual orientado a la realización de tareas } \\
\text { es el que observas cotidianamente en el ambiente escolar. }\end{array}$ & 15 & 75 & 5 & 25 & 0 & 0 \\
\hline 4 & Rara vez se llevan a cabo nuevas ideas en las clases & 4 & 20 & 14 & 70 & 2 & 10 \\
\hline 5 & $\begin{array}{l}\text { En las clases se espera que todos los alumnos hagan el } \\
\text { mismo trabajo, de la misma forma, y en el mismo tiempo }\end{array}$ & 14 & 70 & 4 & 20 & 2 & 10 \\
\hline 6 & $\begin{array}{l}\text { Los docentes inician la clase con frases de motivación en } \\
\text { "valores y virtudes", considerando la realidad del entorno } \\
\text { familiar y/o comunitario }\end{array}$ & 2 & 10 & 14 & 70 & 4 & 20 \\
\hline 7 & $\begin{array}{l}\text { El profesor propone actividades innovadoras para que los } \\
\text { estudiantes las desarrollen }\end{array}$ & 11 & 55 & 9 & 45 & 0 & 0 \\
\hline 8 & $\begin{array}{l}\text { Los métodos de enseñanza en tus clases se caracterizan } \\
\text { por la innovación, la variedad, la participación y la } \\
\text { interacción con los docentes. }\end{array}$ & 15 & 75 & 5 & 25 & 0 & 0 \\
\hline 9 & $\begin{array}{l}\text { Los docentes no se interesan por los problemas de los } \\
\text { estudiantes }\end{array}$ & 7 & 35 & 7 & 35 & 6 & 30 \\
\hline 10 & $\begin{array}{l}\text { En las clases se dan oportunidades para que los estudiantes } \\
\text { expresen su opinión }\end{array}$ & 16 & 80 & 4 & 20 & 0 & 0 \\
\hline 11 & Es el profesor quien decide qué se hace en esta clase & 15 & 75 & 5 & 25 & 0 & 0 \\
\hline 12 & $\begin{array}{l}\text { Se realizan trabajos en grupo (en equipo) con instrucciones } \\
\text { claras y participación del docente. }\end{array}$ & 7 & 35 & 11 & 55 & 2 & 10 \\
\hline 13 & $\begin{array}{l}\text { Los docentes se sienten comprometidos con la gestión y } \\
\text { liderazgo de las autoridades educativas. }\end{array}$ & 11 & 55 & 9 & 45 & 0 & 0 \\
\hline 14 & La ética y los valores se enseñan con el ejemplo & 10 & 50 & 9 & 45 & 1 & 5 \\
\hline
\end{tabular}

Fuente: Encuesta a estudiantes, Centro Educativo "Domingo Savio"

Elaborado Por: Grupo de Investigación

La encuesta a estudiantes demuestra el sentir de los mismos referente a si los directivos escuchan o toman en cuenta las opiniones de ellos responden que si son escuchados a pesar de que cuando se trata de problemas personales no se les realiza un seguimiento ni control de solución, ya que la forma de dirigir va más encaminada a prestar atención a las actividades escolares que las intrafamiliares que como se sabe es una de las mayores falencias en la comunidad educativa de nuestromedio.

Se puede afirmar que embargo los estudiantes están contentos porque en el aula se muestran procesos innovadores de enseñanza por parte de los docentes y se toma en cuenta las propuestas de ellos en la planificación de la temática a trabajar, donde se incorporan temas 
relacionados con valores personales demostrándose interés en el bienestar de las relaciones sociales de los estudiantes lo que se confirma con el buen comportamiento de los docentes tanto en el aula como en cualquier otro lugar de influencia para los estudiantes, esta cualidad es a la que se refiere (Mañu, 2006) cuando dice que el líder transformador es aquel que pone énfasis en los procesos; procura formar a las personas para cambiar la realidad; orienta una relación igualitaria entre él y sus alumnos donde todos aprenden de todos; crea las situaciones propicias para que se dé una educación permanente; desea hacer surgir una conciencia crítica; estimula la cooperación, la solidaridad y la creatividad; y emplea como recursos didácticos los medios de comunicación masiva para analizarlos críticamente junto con sus alumnos.

TABLA 3. DE LA ENCUESTA A PADRES DE FAMILIA

\begin{tabular}{|c|c|c|c|c|c|c|c|}
\hline \multirow{2}{*}{ Orden } & \multirow{2}{*}{ Declaraciones } & \multicolumn{2}{|c|}{ Siempre } & \multicolumn{2}{|c|}{ A veces } & \multicolumn{2}{|c|}{ Nunca } \\
\hline & & f & $\%$ & f & $\%$ & f & $\%$ \\
\hline 1 & $\begin{array}{l}\text { Que tanto conocimiento Ud. posee sobre los } \\
\text { procesos de gestión y liderazgo desarrollados en la } \\
\text { institución donde se educa su hijo }\end{array}$ & 13 & 65 & 5 & 25 & 2 & 10 \\
\hline 2 & $\begin{array}{l}\text { Cataloga Ud. Como buena la relación afectiva entre su } \\
\text { hijo y los demás miembros de la comunidad educativa }\end{array}$ & 3 & 15 & 14 & 70 & 3 & 15 \\
\hline 3 & $\begin{array}{l}\text { La enseñanza impartida en el colegio Domingo Sabio } \\
\text { aporte en el desarrollo integral de su hijo/a }\end{array}$ & 15 & 75 & 5 & 25 & 0 & 0 \\
\hline 4 & $\begin{array}{l}\text { Ayudan en la formación como persona de bien a su hijo/a } \\
\text { la práctica de los valores facilitados en este colegio }\end{array}$ & 4 & 20 & 14 & 70 & 2 & 10 \\
\hline 5 & $\begin{array}{l}\text { Las labores diarias de inter-aprendizaje, que imparten los } \\
\text { maestros del centro educativo, son basados en valores y } \\
\text { buenas relaciones con su hijo/a }\end{array}$ & 14 & 70 & 4 & 20 & 2 & 10 \\
\hline 6 & $\begin{array}{l}\text { Participan los miembros del comité central de padres de } \\
\text { familia en la integración de estrategias metodológicas, } \\
\text { elaboración de reglamentos o códigos de la institución }\end{array}$ & 2 & 10 & 14 & 70 & 4 & 20 \\
\hline 7 & $\begin{array}{l}\text { Existe un buen grado de aceptación por parte de la } \\
\text { comunidad que reconoce en la Capacidad de } \\
\text { Organización de los directivos y el trabajo en equipo de } \\
\text { los docentes de este establecimiento, cuando promueva } \\
\text { verdaderos líderes es: }\end{array}$ & 11 & 55 & 9 & 45 & 0 & 0 \\
\hline 8 & $\begin{array}{l}\text { ¿Cómo considera usted la gestión educativa en cuanto al } \\
\text { trabajo de valores con los adolescentes, de este } \\
\text { establecimiento, desde su creación? }\end{array}$ & 15 & 75 & 5 & 25 & 0 & 0 \\
\hline 9 & $\begin{array}{l}\text { Según su criterio se siente bien con su hijo con el clima } \\
\text { escolar del centro }\end{array}$ & 7 & 35 & 7 & 35 & 6 & 30 \\
\hline
\end{tabular}

Fuente: Encuesta a padres de familia, Centro Educativo "Domingo Savio" Elaborado Por: Grupo de Investigación 
Lo expresado por los padres de familia indica que ellos conocen de forma considerable los procesos de gestión y liderazgo de la institución educativa, lo que los mantiene tranquilos y les da gran confianza referente a la formación de sus hijos, a pesar que en cuanto a las relaciones de afectividad con todos los miembros de la comunidad no es del todo aceptable, ya que los directivos aún no dan una apertura completa a los chicos para que se sientan en gran confianza y expresen todo lo que les afecta, el asunto referente a los valores que se enseñan es el fuerte de la institución gracias a que es una institución fiscomisional.

Se puede afirmar que se da gran énfasis al comportamiento dentro y fuera del aula, esto hace sentir bien a los padres quienes a veces no comparten mucho tiempo con sus hijos y esperan que el colegio cumpla con ese rol que ellos por falta de tiempo no lo hacen como es la formación integra en valores éticos, morales y ciudadanos, otra situación que mantiene tranquilo a los padres es la oportunidad que brinda la institución para que ellos se integren y formen parte de las actividades de liderazgo y de valores así como las sociales con la comunidad educativa tanto así que sus sugerencias son tomadas en cuenta para la construcción de planificaciones y/o proyectos de la institución, esto demuestra que toda la colectividad apoya la gestión del colectivo pedagógico del centro esto lo podemos reafirmar con lo que dice Bedwell G (2004) Ayudar a los padres en el conocimiento de los profesores y sus necesidades y en el desarrollo de una actitud empática y de respeto hacia ellos como personas.

\section{Tabla 4. De la EnTrevista a Directivos}

Nro. Pregunta $\quad$ Respuesta positiva F Respuesta débil F

\begin{tabular}{|c|c|c|c|c|c|}
\hline 1 & $\begin{array}{l}\text { ¿Qué es la comunicación para Ud.? ¿En } \\
\text { qué se diferencia de la información? }\end{array}$ & $\begin{array}{l}\text { Manera de compartir un } \\
\text { criterio entre individuos, } \\
\text { información es dar a } \\
\text { conocer algo }\end{array}$ & 2 & $\begin{array}{l}\text { Grado de } \\
\text { entendimiento entre } \\
\text { personas, información } \\
\text { es algo que solo se } \\
\text { recibe }\end{array}$ & 2 \\
\hline 2 & $\begin{array}{l}\text { ¿El centro educativo, cuenta con un } \\
\text { manual o reglamento que contemple el } \\
\text { cómo, cuándo y quién debe realizar las } \\
\text { tareas? }\end{array}$ & $\begin{array}{l}\mathrm{Si} \text {, el PEI y Código de } \\
\text { convivencia }\end{array}$ & 4 & & 0 \\
\hline 3 & $\begin{array}{l}\text { ¿Cómo actuaría frente a un conflicto entre } \\
\text { la dirección de su centro y el profesorado? }\end{array}$ & $\begin{array}{l}\text { A través del dialogo } \\
\text { como mediador }\end{array}$ & 4 & & 0 \\
\hline 4 & $\begin{array}{l}\text { ¿Cuáles deben ser las características de un } \\
\text { líder educativo? }\end{array}$ & $\begin{array}{l}\text { Mantener buena } \\
\text { comunicación, } \\
\text { responsable y respetuoso }\end{array}$ & 3 & $\begin{array}{l}\text { Democrático, } \\
\text { solidario, honesto }\end{array}$ & 1 \\
\hline 5 & $\begin{array}{l}\text { ¿Cuál es el tipo de liderazgo que } \\
\text { predomina en la dirección, docencia y } \\
\text { alumnado? }\end{array}$ & Democrático & 3 & Responsable & 1 \\
\hline 6 & $\begin{array}{l}\text { ¿Cuáles son los valores institucionales que } \\
\text { busca desarrollar el centro educativo? }\end{array}$ & $\begin{array}{l}\text { Solidarios, honestos, } \\
\text { responsables y } \\
\text { respetuosos }\end{array}$ & 2 & $\begin{array}{l}\text { Capacitados } \\
\text { intelectual y } \\
\text { espiritualmente }\end{array}$ & 2 \\
\hline 7 & $\begin{array}{l}\text { ¿Cuáles son los valores que predominan en } \\
\text { los profesores y alumnos? }\end{array}$ & $\begin{array}{l}\text { Solidaridad, } \\
\text { responsabilidad, respeto }\end{array}$ & 4 & & 0 \\
\hline 8 & $\begin{array}{l}\text { En el caso de existir antivalores, ¿cuáles } \\
\text { son? }\end{array}$ & impuntualidad & 4 & & 0 \\
\hline
\end{tabular}

Fuente: Entrevista a directivos Centro Educativo "Domingo Savio"

Elaborado Por: Grupo de Investigación 
Se observa que tienen claro lo que es la comunicación y que la practican con sus subalternos en pro del buen funcionamiento de la institución, acerca de que si el centro cuenta con un manual o reglamento que contemple las funciones y obligaciones de los miembros vemos que si existe y no solo uno, sino más bien el centro cuento con todos los reglamentos exigidos por el M.E. actualizados y elaborados a su debido tiempo, aunque el PEI no se ha socializado aún con todos los miembros de la comunidad, se observa además que los directivos tienen claro que el dialogo es la mejor vía para solucionar algún conflicto existente entre los docentes o cualquier otro miembro de la comunidad educativa como todo buen líder a quien le deben adornar estas características de tener buena comunicación y ser responsable en su accionar, transmitiendo este tipo de influencia entre sus subalternos logrando que sean responsables, honestos y comunicativos, a más de hacer énfasis en la puntualidad que es uno de los puntos débiles de la comunidad.

\section{Discusión.}

El análisis del centro educativo fiscomisional "Domingo Savio desde la luz de la Gestión Educativa y Liderazgo en Valores conllevo a una excelente reflexión sobre los centros educativos de esta naturaleza los mismos que aparentemente no tienen problemas de ninguna índole, más bien se presentan ante la comunidad como instituciones eficaces e idóneas para la formación de niños, niñas y adolescentes convirtiéndose de esta manera en los más apetecidos por los padres de familia de nuestra localidad. En cuanto a la gestión administrativa en un $50 \%$ se observa en la institución es el rector quien organiza las actividades curriculares o de otra naturaleza, las mismas que las realizan en una reunión general al fin de cada trimestre, cabe señalar que también se da oportunidades para que se organicen grupos de trabajo a los cuales se les da responsabilidades sobre actividades importantes para el buen funcionamiento del centro, por otro lado cuando la necesidad lo amerita son los coordinadores de áreas quienes organizan algún tipo de actividad, especialmente para los eventos culturales, deportivos o científicos que se realizan durante las semanas culturales-deportivas de la institución. Con lo que se puede reflexionar con lo expuesto por Mañú, (2009) quien manifiesta que: definir bien las funciones dentro de un equipo de trabajo es conseguir la eficacia, no basta con limitarse a la eficiencia, sino que deben pretender los resultados que se buscaban; por lo que entregar responsabilidades a coordinadores de área y hacer grupos de trabajo es una idea acertada de los directivos quienes demuestran que existe un clima de liderazgo definido e idóneo donde se avizora el marco del respeto y valores para con todos los miembros de lainstitución.

En cuanto a los aspectos que se toman en cuenta para medir el tamaño de la organización, se evidencia en un $100 \%$ que se mide tomando en cuenta los resultados obtenidos en la institución, lo que concuerda con los objetivos planteados en los documentos del meso currículo los cuales apuntan a que se cumplan metas ya sean a mediano y largo plazo (PEI, C.E.G.B. "Domingo Savio", 2012), es decir el tamaño de la organización depende de los resultados obtenidos después de cada análisis del funcionamiento y crecimiento de la 
misma, los directivos realizan reuniones periódicas donde tratan temas referentes a la imagen que el centro brinda a la comunidad como la forma de captar mayor clientela, esto se lo observa por el nivel de aceptación que año a año aumenta entre los habitantes de la localidad.

No se debe dejar pasar por alto el indicar que el 100\% de las tareas, obligaciones y demás responsabilidades de los miembros de la institución se encuentran estipuladas en el código de convivencia instrumento este que se convierte en el manual de normas y que rige todo el funcionamiento ordenado del centro, a pesar que no existe un reglamento interno individual que rija las obligaciones y responsabilidades de los miembros del centro, este manual es muy práctico y permite organizar muchos aspectos legales y de interrelación entre todos los miembros de la comunidad, lo que se puede reforzar con lo expresado por Mañu, $(2009,36)$ El Código de Convivencia tiene como objetivo la adecuación de los estilo de convivencia institucional a los requerimientos de la sociedad actual, se plantea como un proyecto flexible y capaz de retroalimentarse creativamente, a través del aporte y cuestionamiento de todos los integrantes de la comunidad educativa.

Lo que confirma el buen nivel de comprensión por parte de los directivos en cuanto a manejar un documento que permita el control y buen desempeño de cada individuo relacionado con el centro, además es importante saber que en este instrumento se hace mucho énfasis al cultivo de los valores y principios cristianos que rigen al centro educativo. A pesar que el código indica con claridad las obligaciones y sanciones a quienes incumplan con la reglamentación, es importante saber que los directivos manejan una política de delegar y permitir que cada departamento realice las actividades que le corresponde con lo que cada miembro del centro se convierte en alguien importante para el engranaje y buen funcionamiento del mismo, especialmente en la solución de situaciones conflictivas que merecen un estudio más detenido tanto de su génesis como de su solución para que no se repitan en otra ocasión, esto lo podemos contrastar con lo expuesto por Bilikopf (2012) La participación en la toma de decisiones puede mejorar la calidad y la aceptación de las decisiones, fomenta la motivación y la autoestima de los trabajadores y mejora las relaciones interpersonales con los empleados. Pero no es siempre fácil el delegar.

Ya que aquí delegar es una actividad común de los directivos y personal responsable. La dirección del centro promueve a la excelencia académica en un 100\% como el factor primordial para el buen funcionamiento y éxito del mismo, sin dejar de lado el trabajo en equipo puesto que muchas de las acciones de este se realizan en grupos dirigidos y coordinados por los directores de área quienes organizan actividades de todo tipo de naturaleza involucrando a estudiantes y padres de familia, lo que nos lleva a aceptar que la institución está organizada tomando en cuenta aspectos neurálgicos que conllevan a la excelencia académica y valoran mucho la participación de los padres de familia. Es mucho lo que se puede decir de las habilidades que se requieren para dirigir una institución 
educativa, ya que en un $100 \%$ observamos que son innatas, vemos en porcentajes menores que estas se pueden adquirir estudiando y capacitándose continuamente, para lo cual la institución debe implementar una política interna de orientación y capacitación a los docentes que en algún tiempo tomaran la posta para seguir dirigiendo, es muy escuchado que un líder nace, no se hace, pero un líder educativo puede formarse con la capacitación permanente y la buena dirección y acompañamiento de quienes le anteceden en esta responsabilidad, es por eso que en la encuesta a directivos referente a este tema resalta a la adquisición a partir de la experiencia como una de las respuestas más comunes refiriéndose a que los nuevos líderes se apoyan en la experiencia de suspredecesores.

Es hora de tocar cuales son los organismos presentes en la institución, en las repuestas observamos que la institución tiene todos los organismos bien estructurados, tanto los de dirección o gestión los que ocupan un 100\%, a mas de académicos y administrativos, los mismos que cumplen funciones particulares de acuerdo a su naturaleza evitando el cruce de responsabilidades lo que hace que la gestión del centro se vuelva más eficiente y concuerdo con lo expresado con Martín,( 2006), los órganos colegiados de gobierno y de coordinación docente de los centros públicos son: el consejo escolar, el claustro de profesores y otros órganos de coordinación docente; los órganos de gobierno unipersonales son el director y cada uno de los miembros de su equipo (jefe de estudios y secretarios) y cualquier otro órgano que, en su caso, establezca la legislación.

Se puede concluir que el centro posee todos los órganos colegiados y también hacen funcionar los órganos unipersonales los mismos que cumplen con los lineamientos estipulados en la misión y visión del centro. Es así que el claustro de profesores hace énfasis en coordinar las actividades de enseñanza y aprendizaje que se propongan a los alumnos como función principal de este organismo, aunque también se debe dedicar a llevar a cabo la evaluación o seguimiento global del grupo de alumnos ya que ellos son la razón de ser del centro por lo que se les da mayor atención, proponiendo estrategias de recuperación ante situaciones académicas o conflictivas que se den durante el proceso educativo, dentro de estas actividades se puede resaltar a las convivencias donde se trabaja mucho en la formación moral de los estudiantes, cabe señalar que los padres de familia también son involucrados en este tipo de actividades donde pueden compartir con sus hijos actividades de reflexión, estudio de los principios cristianos y otras actividades lúdicas que integren la familia y rescaten el amor y confraternidad que son base de la convivencia cristiana.

El centro tiene organizada la parte didáctica desde el vicerrectorado quien ha conformado los diferentes departamentos o áreas que se encargan de toda la parte académica desde la orientación a los docentes hasta la revisión de planificaciones, evaluaciones y proyectos presentados ya sean por docentes como por los estudiantes, se realizan reuniones periódicas cada semana por departamento y cada mes entre los directores a área y el vicerrector para diagnosticar el avance académico e implementar los planes de mejora o 
recuperación didáctica, en esta actividad juegan un papel fundamental los padres de familia quienes son invitados a participar en los planes de recuperación, y control de estas actividades en los hogares. La institución en cuanto a organización de instrumentos didácticos posee una gran fortaleza ya que cuenta con todo tipo de material de planificación educativa, a pesar que en ellos no presentan actividades de formación extra curricular que conlleven al buen uso del tiempo libre y por ende a la formación integral de los estudiantes, lo que avizora que esta organización necesita una reestructuración de los mismos ya que toda institución que tenga su plan estratégico, plan operativo y realice reingeniería de procesos se la considera a la vanguardia en cuanto a educación se refiere, esto se lo puede considerar a la luz de lo expuesto por Lowenthal, (2001) una institución educativa poseer los siguientes instrumentos de gestión: El código de ética, el plan estratégico, el POA, el reglamento interno, el PEI, el manual de organización; manejar metodologías de reingeniería de procesos que se la conoce como: El pensamiento nuevo y el rediseño fundamental de los procesos operativos y la estructura organizacional, orientada hacia las competencias esenciales de la organización, para lograr mejoras dramáticas en el desempeño organizacional.Lo que determina la gran estructura didáctica de este centro educativo, cabe recordar que es la mayor fortaleza del mismo, y gracias a esto mantiene una gran aceptación de la comunidad esmeraldeña.

Al hablar del sentir del cuerpo docente en un $75 \%$ se puede afirmar que las relaciones docentes-autoridades se manejan bajo el clima de respeto y valores bien arraigados, a pesar que los docentes no estén del todo de acuerdo en propuestas de nuevas políticas de enseñanza, ya que no son afines a la improvisación o implantación de acciones no probadas anteriormente, los valores cumplen un rol protagónico refiriéndonos a la formación integral de los estudiantes, estos se complementan con los principios cristianos regentados por el sistema preventivo de Don Bosco el cual gira en su máxima idea de formar excelentes ciudadanos y buenos cristianos, se puede apreciar que hay una relación armónica entre docentes y directivos respecto a las políticas de capacitación y liderazgo a pesar que un $25 \%$ de docentes no están del todo conforme con la manera como se maneja el asunto de los nuevos métodos de enseñanza, una de las debilidades observadas es la falta de confianza por parte del personal en la integración para la toma de decisiones, ya que esta función de los directivos es realizada por personas externas a la institución como lo son los representantes de la iglesia, quienes no permanecen el tiempo suficiente en la institución para conocer la realidad veraz y objetiva de la misma, y a veces toman decisiones erradas con relación al accionar o funcionamiento del centro.

Cabe resaltar que los departamentos encargados de la integración de los miembros de la comunidad educativa, cumplen a cabalidad su rol en cuanto a la planificación de actividades de integración de los padres de familia, logrando de esta manera que ellos se identifiquen de forma directa con el andamiaje del proceso de formación de sus hijos, sin embargo esta integración solo se la realiza en lo referente a actividades de confraternidad, 
o de carácter religioso, en cuanto a las actividades relacionadas con la parte curricular o de gestión académica los padres se sienten aislados ya que no ocurre ni la socialización de los documentos de gestión como el PEI mucho menos se toma en cuenta sus opiniones para la construcción de los mismos. También se avizora que los docentes propician su liderazgo sin rechazar o cuestionar las decisiones de los directivos a pesar de no estar muy de acuerdo en que las delegaciones de actividades no se manejan como ellos lo esperan ya que la parte administrativa financiera no puede sr manejada desde la institución a más de que no existe una política de rendición de cuentas por que la manejan directamente los representantes de la iglesia.

Los estudiantes tienen un criterio muy firme en cuanto al lugar que ocupan como la razón de ser de la institución, están claros en un $75 \%$ en que todo el andamiaje educativo debe girar en torno a ellos; sin embargo es preocupante saber que los directivos no toman muy en cuenta las opiniones de ellos, o si bien los escuchan no se ven los resultados de las sugerencias hechas por ellos ya que la forma de dirigir va más encaminada a prestar atención a las actividades escolares que las intrafamiliares que como se sabe es una de las mayores falencias en la comunidad educativa de nuestro medio; sin embargo los estudiantes están contentos porque en el aula se muestran procesos innovadores de enseñanza por parte de los docentes y se toma en cuenta las propuestas de ellos en la planificación de la temática a trabajar, realizándose actividades motivadoras dentro y fuera del aula, los docentes tienen claro que el aula de clases no solamente es un salón, sino todo ambiente propicio donde se pueda educar con el ejemplo e impartiendo valores, con la finalidad de formar los nuevos líderes responsables del futuro del país.

Para los padres de familia en un $80 \%$ el clima escolar del centro es agradable, pero no del todo idóneo, ya que observan que se podría realizar más actividades que involucren a sus hijos en situaciones relacionados con valores y liderazgo, piensan que se les debería tomar más en cuenta en cuanto a la socialización de reglamentos o planes curriculares ya que en conjuntos se podrían realizar proyectos de mejoras en bienestar de los estudiantes. Afirman que conocen de forma considerable los procesos de gestión y liderazgo de la institución educativa, lo que los mantiene tranquilos y les da gran confianza referente a la formación de sus hijos, a pesar que en cuanto a las relaciones de afectividad con todos los miembros de la comunidad no es del todo aceptable, ya que los directivos aún no dan una apertura completa a los chicos para que se sientan en gran confianza y expresen todo lo que les afecta, para ello proponen se realicen más actividades de integración entre autoridades y estudiantes, por que como se sabe son los docentes quienes más comparten con los chicos y es a ellos quienes los chicos les confían sus problemas e inquietudes, sin embargo quienes tienen que legislar sobre alguna falta o sanción son las autoridades y quienes a veces no conocen la génesis de los problemas. Cabe destacar que el asunto referente a los valores que se enseñan es el fuerte de la institución gracias a que es una institución fiscomisional, se da gran énfasis al comportamiento dentro y fuera del aula, esto hace sentir bien a los 
padres quienes a veces no comparten mucho tiempo con sus hijos y esperan que el colegio cumpla con ese rol que ellos por falta de tiempo no lo hacen como es la formación integra en valores éticos, morales y ciudadanos, otra situación que mantiene tranquilo a los padres es la oportunidad que brinda la institución para que ellos se integren y formen parte de las actividades relacionadas con liderazgo y valores como en las de carácter social con la comunidad educativa tanto así que sus sugerencias son tomadas en cuenta sirviendo esto de gran apoyo a la institución, esto demuestra que toda la colectividad apoya la gestión del colectivo pedagógico del centro.

Los padres reconocen el buen trabajo realizado por los miembros de la institución desde su creación hasta la actualidad y comparten el sentir de la sociedad de que el centro es un referente local en cuanto a la formación didáctico-pedagógica como a la formación en valores y liderazgo lo que se observa por la gran acogida que tiene el centro en cuanto a la población estudiantil, y se comprometen a contribuir con el buen funcionamiento del mismo.

Finalmente los directivos en un 100\% aseguran que en la institución existe una excelente vía de comunicación, entre todos los miembros de la comunidad, tanto así que son tomadas en cuenta las sugerencias tanto de padres como estudiantes y docentes, rescatando la existencia de un agradable clima escolar, están claros en que los valores que identifican a los miembros del centro son la responsabilidad, honradez y comprometimiento mutuo del mejoramiento de la institución, están claros que falta trabajar un poco con la puntualidad y cumplimiento de la socialización de documentos y reglamentos, para lo cual están implementando un plan de contingencia en donde su primordial aspecto será la socialización e integración de actividades curriculares con los padres de familia.

\section{Conclusiones.}

- Los referentes teóricos sobre gestión educativa, liderazgo educativo gestión de la calidad en valores investigados en comparación con los del centro educativo fiscomisional Domingo Savio se interrelacionan de manera efectiva lo que se evidencia con su planificación organizacional bien estructurada, basada en el sistema preventivo de Don Bosco en la que destaca la formación de la conducta del ser como eje transversal de toda suformación.

- Los miembros de la comunidad educativa no poseen conocimiento pleno en cuanto a los instrumentos de gestión, aún así desarrollan sus actividades con alto grado de responsabilidad, demostrando de esta manera su rol de buenos líderes en la función que desempeñan. 
- La práctica de valores y liderazgo es realizada entre todos los miembros de la comunidad, tomando en cuenta las opiniones y respetando las ideas de quienes forman parte de la institución.

- Los miembros de la comunidad participan en las actividades del centro de forma directa, aunque las conclusiones finales de construcción de documentos no son socializadas por parte de los directivos a los demás miembros quienes desconocen el norte a seguir en cuanto a lineamientos curriculares.

- Las actividades de integración (convivencias, escuelas para padres) entre padres y estudiantes son uno de los puntos más acertados de la planificación institucional, a pesar de que se realizan una o dos veces al año, estas proporcionan la integración de la comunidad educativa.

- La no inclusión en los documentos de gestión de actividades extra curriculares, las escasas alternativas de ocupación del tiempo libre y la falta de lugares de esparcimiento o actividades recreativas no permiten el desarrollo integral de destrezas, habilidades y talentos no permiten que se complete el proceso de formación en valores y convivencia de los estudiantes.

\section{Referencias bibliográficas.}

Aguerrondo, I. (1991). El planeamiento como instrumento de cambio. Argentina: TROQVEL. Obtenido de http://www.terras.edu.ar/biblioteca/12/ECPI_Aguerrondo_Unidad_2.pdf

Amat , J. (2007). Control de gestion: una perspectiva de direccion. Barcelona: EDICIONES GESTION 2000.

Calderón, A. (2015). Gestión, liderazgo y valores en la Administración de la Unidad Educativa Mariscal Sucre, de Guayaquil, provincia del Guayas; durante el año 2013. Tesis de Posgrado Universidad Técnica Particular de Loja, 162. Loja, Ecuador. Obtenido de http://dspace.utpl.edu.ec/bitstream/123456789/13337/1/Calderon_Morales_Ana_Ma ria.pdf

Castro, P. (2014). Toma de Decisiones Asertivas para una Gerencia efectiva. Tesis de Pregrado Universidad Militar Nueva Granada. Colombia. Obtenido de 
http://repository.unimilitar.edu.co/bitstream/10654/11746/1/TOMA\%20DE\%20DE CISIONES\%20ASERTIVAS\%20PARA\%20UNA\%20GERENCIA\%20EFECTIV A.pdf

Fernandez , B., Morales , I., \& Portal , J. (2004). Sistema de influencias para la formación integral de los egresados de los centros de Educación Médica Superior. Educacion Medica superior, 18(2). Obtenido de http://scielo.sld.cu/scielo.php?script=sci_arttext\&pid=S0864-21412004000200002

Guachamin, S. (10 de mayo de 2012). Gestión, Liderazgo y Valores en la Administración del Centro Educativo “Club Árabe Ecuatoriano” Durante elAño Lectivo 2010-2011. Tesis de prosgrado UTEPL. Loja, Ecuador. Obtenido de http://dspace.utpl.edu.ec/bitstream/123456789/6382/1/TESIS.pdf

Morales , G. (2012). Ética para todos : cómo educar hoy en ética, valores y moral. Guayaquil: Eduquil.

Palacios , M. (2011). Gestión del liderazgo y valores en la Unidad Educativa Particular Experimental Bilingüe Rosa de Jesús Cordero, de la ciudad de Cuenca, durante el año 2010-2011. tesis Posgrado Universidad Técnica Partiular de Loja(173). Ecuador. Obtenido de http://dspace.utpl.edu.ec/bitstream/123456789/3091/1/Tesis\%20de\%20Palacios\%20 Moreno\%20Maria\%20Victoria.pdf

Peréz , E. (2014). Psicología institucional. Mar del Plata: Edulp. Obtenido de http://sedici.unlp.edu.ar/bitstream/handle/10915/42516/Documento_completo.pdf?s equence $=1$

Quintana , M., \& Moreno, C. (2007). Organizacion y direccion de centros educativos innovadores: el ce ntro educativo versatil. Madrid, España : S.A. MCGRAW-HILL.

Rodriguez, F., \& Bravo, L. (1991). Indicadores de productividad en la empresa. Venezuela: Nuevos Tiempos. Obtenido de http://scioteca.caf.com/bitstream/handle/123456789/863/Indicadores\%20de\%20cali dad\%20y\%20productividad\%20en\%201a\%20empresa.PDF 


\section{Para citar el artículo indexado.}

Puyol J., Alcívar E., López M. \& Guamán R. (2018). Análisis de la gestión del liderazgo y valores en la administración de las unidades educativas. Revista electrónica Ciencia Digital 2(1), 214-232. Recuperado desde:

http://www.cienciadigital.org/revistascienciadigital2/index.php/CienciaDigital/article/view $\underline{/ 15 / 15}$

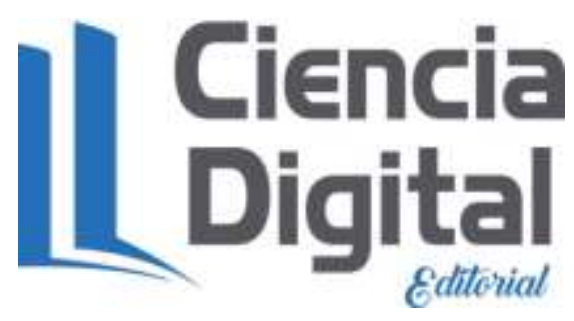

El artículo que se publica es de exclusiva responsabilidad de los autores y no necesariamente reflejan el pensamiento de la Revista Ciencia Digital.

El articulo queda en propiedad de la revista y, por tanto, su publicación parcial y/o total en otro medio tiene que ser autorizado por el director de la Revista Ciencia Digital.
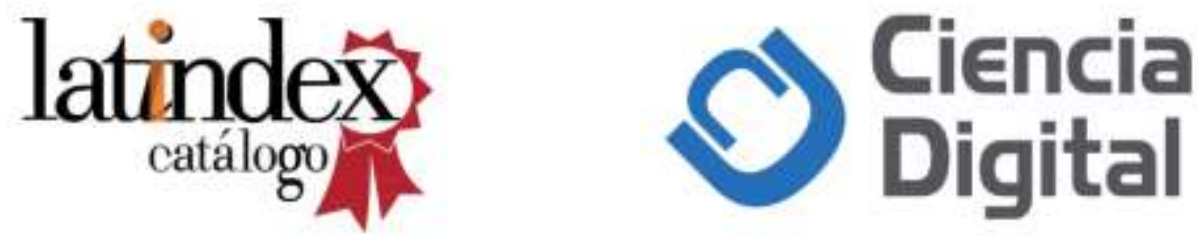